\title{
Alismatales from the upper and middle Araguaia river basin (Brazil)
}

\author{
SAMANTHA KOEHLER ${ }^{1,3}$ and CLAUDIA PETEAN BOVE ${ }^{2}$
}

(received: October 30, 2002; accepted: March 25, 2004)

\begin{abstract}
Alismatales from the upper and middle Araguaia river basin (Brazil)). The present study deals with a survey of the order Alismatales (except Araceae) in the upper and middle Araguaia River region located between the states of Mato Grosso and Goiás, Brazil. Field expeditions were carried out during the rainy and dry seasons. The route covered approximately $2,000 \mathrm{~km}$ and 41 aquatic environments were visited. Thirteen taxa, representing the families Alismataceae (nine), Hydrocharitaceae (three) and Najadaceae (one) were identified. Keys for the identification of families and species in field, brief diagnoses, schematic illustrations and relevant comments were elaborated based on field observations as well as on the analysis of the specimens collected.
\end{abstract}

Key words - Alismatales, aquatic plants, Araguaia river, Brazil, hydrophytes

RESUMO - (Alismatales da bacia do alto e médio rio Araguaia (Brasil)). Realizou-se o levantamento de espécies da ordem Alismatales (exceto Araceae) ocorrentes na região do alto e médio rio Araguaia, entre os estados de Mato Grosso e Goiás, Brasil. As expedições para coleta de material, ocorridas tanto na época de chuvas quanto na seca, totalizaram cerca de $2.000 \mathrm{~km}$ percorridos, abrangendo 41 ambientes aquáticos. Foram identificados treze táxons pertencentes às famílias Alismataceae (nove), Hydrocharitaceae (três) e Najadaceae (uma). Foram elaboradas chaves para identificação em campo das famílias e espécies, descrições breves, ilustrações esquemáticas e comentários relevantes, baseados em dados levantados em campo e através da análise do material coletado.

Palavras-chave - Alismatales, Brasil, hidrófitas, plantas aquáticas, rio Araguaia

\section{Introduction}

Brazil has the largest hydrographic net of the world, estimated as $6,950 \mathrm{~km}^{2}$, representing the largest hydric supply of South America (Tundisi \& Barbosa 1995). Plants encountered growing in permanent or seasonally wet environments, whose photosynthetic parts are constantly or at least for several months each year in contact with water are defined by Cook (1996) as vascular aquatic plants. The aquatic flora plays an important role on the ecology of aquatic ecosystems. It affects the water chemistry by the assimilation of nutrients and noxious substances (Esteves \& Camargo 1986) and provides favorable conditions to innumerous organisms, such as fishes, aquatic arthropods, mollusks and periphyton (Esteves 1988, Pott \& Pott 1997). Aquatic plants also constitute the base of an extensive food chain (Esteves 1988, Niering 1985) and some species may even be used as ornamental plants, soil fertilizers or for

1. Universidade Estadual de Campinas, Instituto de Biologia, Departamento de Botânica, Caixa Postal 6109, 13083-970 Campinas, SP, Brasil.

2. Universidade Federal do Rio de Janeiro, Museu Nacional, Departamento de Botânica, Quinta da Boa Vista, 20940-040 Rio de Janeiro, RJ, Brasil. cpbove@ibpinet.com.br

3. Corresponding author: koehler@unicamp.br cattle nourishment (Berg 1984, Joyce 1990). However, the excessive growth of some species may interfere with the functioning of hydroelectric turbines, favor the proliferation of mosquitoes, reduce water oxygen concentration, and prevent navigation (Esteves 1988, Palombo \& Pereira 1992).

Despite the great diversity of hydrophytes in Brazil (Hoehne 1948), there is still a lack of studies on the ecological and systematic aspects of Brazilian aquatic plants (Pott et al. 1992). Besides the work of Hoehne (1948) on aquatic plants of Brazil, there are studies, generally taxonomically and/or geographically restricted, developed for the Amazonian region (Albuquerque 1981) and for the states of Goiás (Wanderley 1989), Mato Grosso (Prado et al. 1994, Schessl 1997) Mato Grosso do Sul (Guimarães 1999; Pott 1993, Pott \& Pott 1997, Pott et al. 1989, 1992, Pott \& Cervi 1999, Pott \& Pott 2000 [Pantanal]), Minas Gerais (Pedralli et al. 1993a, b), Rio de Janeiro (Henriques et al. 1988, Bove et al. 2003), Rio Grande do Sul (Almeida-Rego 1988, Irgang et al. 1984, Irgang \& Gastal 1996, Pedralli, 1981), Santa Catarina (Pedersen \& Klein 1976) and São Paulo (Abreu 1981, Faria 1998).

The order Alismatales, with roughly 3,320 species, consists mostly of aquatic or wetland plants and is represented in Brazil by approximately 32 genera and 11 families (Haynes \& Holm-Nielsen 1985, Judd et al. 
1999, Les \& Haynes 1995). A generic treatment of the order in the Neotropics has been published by Haynes \& Holm-Nielsen (1985), besides surveys and taxonomic treatments on the families Alismataceae (Almeida Rego 1988, Guimarães 1999, Haynes \& Holm-Nielsen 1994, Rataj 1978), Hydrocharitaceae (Cook 1985, Cook \& Urmi-König 1984a, b), Limnocharitaceae, included in Alismataceae in this paper (Haynes \& Holm-Nielsen 1992), and Najadaceae (Lowden 1986).

Despite the fact most studies of aquatic plants published so far for the central region of Brazil are geographically and/or taxonomically restricted, there are still innumerous aquatic habitats in this region that are poorly known. This assertion was corroborated by the discovery of a new species of Hydrocharitaceae, Egeria heterostemon S. Koehler \& C.P. Bove, during the development of this study (Koehler \& Bove 2001). The purpose of this study is to contribute to the knowledge of the Brazilian aquatic flora presenting a survey of the order Alismatales of the upper and middle Araguaia River region.

\section{Material and methods}

The study area is located on the Araguaia - Tocantins depression, in the states of Mato Grosso and Goiás, Brazil. The climate, warm and wet, is composed of two main seasons: a dry one from June to November followed by a rainy season from December to May (IBGE 1989). The vegetation is characterized mainly by the Cerrado type, rather devastated, and by riparian forests and aquatic ecosystems, hitherto well preserved. Field expeditions were undertaken in 1997 (May-June and October) and in 1999 (November), covering the rainy and dry seasons. Specimens were collected in 41 different aquatic environments along the roads indicated in figure 1 , covering an extension of approximately $2,000 \mathrm{~km}$. The sites visited consisted mainly of permanently and temporally undrained floodplains.

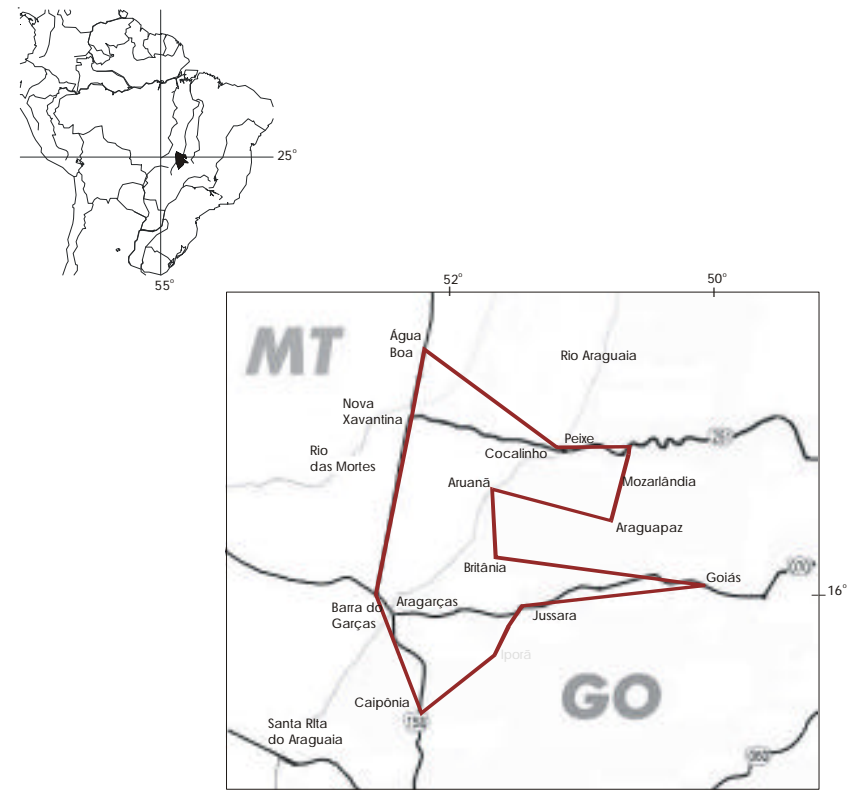

Figure 1. Geographical location of the study area.

Species identifications were based on the taxonomic treatments available and were complemented by comparisons with herbaria material. The material was deposited in the herbarium of the Museu Nacional do Rio de Janeiro (R) and duplicates were sent to the to herbaria of the Universidade de São Paulo (SPF) and to Embrapa - Pantanal (CPAP). Keys for the identification of the families and species in the field, brief diagnoses, schematic illustrations and relevant ecological and morphological comments were elaborated based on an analysis of specimens collected, as well as on field observations.

\section{Results and Discussion}

In the treatment that follows thirteen taxa were recognized.

\section{Key to families of Alismatales of the upper and middle Araguaia river region}

1. Submersed plants

2. Leaves sessile, 1-veined, margins serrulate

3. Leaves with sheathing base, margins with visible serrulations; flowers lacking a perianth, inconspicuous

Najadaceae

3. Leaves without sheathing base, margins without visible serrulations; flowers conspicuous, with white perianth Hydrocharitaceae

2. Leaves petiolate, multi-veined, margins entire

4. Leaves up to $10 \mathrm{~cm}$ long; flowers white Alismataceae

4. Leaves longer than $10 \mathrm{~cm}$; flowers yellow Hydrocharitaceae

1. Emersed plants Alismataceae 
Alismataceae Vent.

Herbaceous, annual or perennial, glabrous or pubescent, lactiferous. Stems corm-like, rhizomatous or stoloniferous. Leaves submersed, floating or emergent, basal or alternate, petiolate with a sheathing base; submersed leaves linear, sessile; emergent and floating leaves petiolate, the blade linear to lanceolate, ovate to rhomboid, pluriveined, with or without pellucid marks, the margins entire. Inflorescences verticillate, paniculate, racemose, spicate or umbellate, without a subtending spathe, with whorled or separate bracts. Flowers hypogynous, perfect or imperfect, pedicellate, perianth actinomorphic, of 6 free segments in 2 series: sepals 3 , green, persistent; petals 3, larger than sepals, white to yellow sometimes with purple spots at the base, delicate, deciduous; stamens 3 or numerous, free, staminodes occasionally present; carpels 3,6 or more, free or basally fused, 1-loculed, ovules 1-2 or numerous of basal or laminar placentation, styles terminal or lateral sometimes very short or absent, stigma linear. Fruits achenes or follicles, numerous, compressed or terete, often winged, longitudinally costate and glandular, sometimes with a terminal or horizontal beak. Seeds U-shaped.

Key to species of Alismataceae of the upper and middle Araguaia river region

1. Floatting leaves sagittate

5. Sagittaria guayanensis

1. Emersed leaves linear, lanceolate or ovate

2. Plants delicate, to $25 \mathrm{~cm}$ tall

3. Pellucid lines on leaf blades present

3. Pellucid lines on leaf blades absent

1. Echinodorus bolivianus

2. Plants stout, taller than $40 \mathrm{~cm}$

4. Ovules of basal placentation

5. Floral bracts subulate, usually longer than the pedicels

5. Floral bracts lanceolate, usually shorter than the pedicels

6. Submersed leaves absent; flowers perfect 4. Echinodorus tenellus

6. Submersed leaves present; flowers, at least the lower, imperfect.

4. Ovules of laminar placentation

7. Blades ovate to orbicular, as long as wide

7. Blades at least twice longer than wide

8. Leaves floating; carpels 3; staminodes 3

8. Leaves erect; carpels and staminodes numerous

3. Echinodorus subalatus

2. Echinodorus longipetalus 6. Sagittaria rhombifolia 7. Hydrocleys nymphoides 8. Hydrocleys parviflora 9. Limnocharis laforestii

1. Echinodorus bolivianus (Rusby) Holm-Niels., Brittonia 31(2):276. 1979. Type: BOLIVIA: Reyes, White 1540 (lectotype NY, fide Haynes \& HolmNielsen 1994; isolectotypes, GH, K, NY, US).

Figure 2A.

Plants delicate, annual, glabrous, stoloniferous, to $15 \mathrm{~cm}$ tall. Submersed leaves present; emersed leaves petiolate, $10 \mathrm{~cm}$ long, $1.0-1.5 \mathrm{~cm}$ wide, the blade lanceolate with a sheathing base, pellucid markings present as lines. Inflorescence umbelliform with only one floral whorl or racemose with two whorls, erect, up to $25 \mathrm{~cm}$ long, without vegetative proliferations. Bracts triangular, basally united, coarse with thin margins, $0.5 \mathrm{~cm}$ long, $0.1-0.2 \mathrm{~cm}$ wide. Flowers perfect, 2-6, $1 \mathrm{~cm}$ diam.; sepals appressed, $0.3 \mathrm{~cm}$ long; petals clawed, 0.3-0.4 cm long; stamens $9,0.1 \mathrm{~cm}$ long, carpels 15-20. Fruits not observed.
Common name: "dwarf amazon swordplant", "erva do pântano" (Haynes \& Holm-Nielsen 1994, Pott \& Pott 2000).

Specimens examined: BRAZIL: GoIÁs: Estr. Jussara-Aragarças a 100 km de Jussara, 9-X-1997 (fl.), C.P. Bove et al. 238. (R, SPF).

Habitat: amphibious or submersed plant, growing in still or running water up to $50 \mathrm{~cm}$ deep. Rare.

Distribution: from Central America to southern South America. In Brazil, in Distrito Federal, Goiás, Mato Grosso, Mato Grosso do Sul, Minas Gerais, Pará, Paraná, Rio Grande do Sul, Rio de Janeiro, Santa Catarina and São Paulo (Haynes \& Holm-Nielsen 1994).

2. Echinodorus longipetalus Micheli, Monogr. Phan. 3:60. 1881. Type: PARAGUAY: Cordillera de Peribebuy, Patiño Cué, Balansa 570 (lectotype K, fide Rataj 1969; isolectotypes, $\mathrm{G}, \mathrm{K})$.

Figure 2B. 
Plants stout, perennial, glabrous, to $200 \mathrm{~cm}$ tall. Submersed leaves absent; emersed leaves petiolate, $40-120 \mathrm{~cm}$ long, $10-15 \mathrm{~cm}$ wide, with a sheathing base $5-15 \mathrm{~cm}$ long, the blade lanceolate, pellucid markings reticulate. Inflorescence racemose, erect or decumbent, overtopping the leaves, without vegetative proliferations, 2-10 whorls with 3-15 flowers each. Bracts lanceolate with an attenuate apex, coarse, $2 \mathrm{~cm}$ long. Flowers perfect; sepals erect, covering the mature fruit, $1-1.5 \mathrm{~cm}$ long, $0.5-1 \mathrm{~cm}$ wide; petals $2 \mathrm{~cm}$ long, $1.5-2 \mathrm{~cm}$ wide; stamens numerous, $0.5 \mathrm{~cm}$ long, carpels numerous. Fruits flattened, dorsally keeled, $0.3 \mathrm{~cm}$ long, the beak lateral, horizontal.

Common name: "narrow-leaved Amazon swordplant", "chapéu de couro" (Haynes \& HolmNielsen 1994, Pott \& Pott 2000).

Specimens examined: BRAZIL: GoIÁs: Estrada Jussara-Aragarças, a $100 \mathrm{~km}$ de Jussara, 26-V-1997 (fl.), C.P. Bove et al. 158, 159 (R); Estrada AruanãBritânia, a 33 km de Aruanã, 14-X-1997 (fl.), C.P. Bove et al. 295 (R); Estrada Jussara-Britânia, ca. de 40 km do trevo, 14-XI-1999 (fl., fr.), C.P. Bove et al. 577 (R, SPF). Mato Grosso: Estrada Água Boa-Cocalinho, a 144 km de Água Boa, 12-X-1997 (fl., fr.), C.P. Bove et al. 272 (R, SPF).

Habitat: emergent plant, growing in still water up to $50 \mathrm{~cm}$ deep. Commonly found around the edges of marshes, usually presenting a high local abundance.

Distribution: Suriname and from central Brazil (states of Goiás, Mato Grosso, Mato Grosso do Sul, Minas Gerais and São Paulo) to eastern Paraguay (Haynes \& Holm-Nielsen 1994, Pott \& Pott 2000).

3. Echinodorus subalatus (Mart.) Griseb. subsp. subalatus, Cat. pl. Cub. p.218. 1866. $\equiv$ Alisma subalatum Mart., Syst. veg. 7(2):1609. 1830. Type: BRAZIL: s. loc., C.F.P. Martius 150 (lectotype M, fide Rataj 1971; isolectotype MO).

Figure 2C-D.

Plants stout, perennial, glabrous, $40-100 \mathrm{~cm}$ tall. Submersed leaves absent; emersed leaves petiolate, 23-55 cm long, 1-8 $\mathrm{cm}$ wide, with a sheathing base $6-15 \mathrm{~cm}$ long, the blade lanceolate, pellucid markings present as lines. Inflorescence racemose or paniculate, erect, 50-60 cm long, with or without vegetative proliferations, 5-6 whorls with 5-10 flowers each. Bracts subulate, coarse, with delicate margins, $0.5-2 \mathrm{~cm}$ long. Flowers perfect; sepals reflexed, $0.5 \mathrm{~cm}$ long, $0.5 \mathrm{~cm}$ wide; petals $1 \mathrm{~cm}$ long, $0.5-1 \mathrm{~cm}$ wide; stamens 20 , $0.4 \mathrm{~cm}$ long, carpels numerous. Fruits terete, unkeeled, glandular, 0.1-0.2 cm long, the beak terminal, erect.
Common name: "longstyled toadspoon", "chapéu de couro miúdo" (Haynes \& Holm-Nielsen 1994, Pott \& Pott 2000).

Specimens examined: BRAZIL: GoIÁs: Estrada Jussara-Aragarças, a 3 km de Jussara, 9-X-1997 (fl., fr.), C.P. Bove et al. 246 (R, SPF); Estrada AruanãBritânia, a 10 km de Britânia, 14-XI-1999 (fl.), C.P. Bove et al. 586 (R); Estrada para Peixe (GO-334), a $39 \mathrm{~km}$ da GO-164, 16-XI-99 (fl.), C.P. Bove et al. 624 (R). Mato Grosso: Estrada Água Boa-Cocalinho, a $100 \mathrm{~km}$ de Água Boa, 12-X-1997 (fl., fr.), C.P. Bove et al. 264 (R).

Observations: we found specimens with vegetative proliferating inflorescences (C.P. Bove et al. 246), a character not recorded in previously taxonomic treatments of this species (Haynes \& Holm-Nielsen 1994, Rataj 1978).

Habitat: emergent plant, growing in still water up to $50 \mathrm{~cm}$ deep; however, it may be found in the middle of rivers. Commonly found around the edges of marshes and as a dominant species in polluted environments.

Distribution: from Central America to south-central Brazil, states of Amazonas, Bahia, Ceará, Goiás, Maranhão, Mato Grosso, Mato Grosso do Sul, Minas Gerais, Paraná, Rio Grande do Norte, Rio de Janeiro, Roraima, Santa Catarina and São Paulo (Haynes \& Holm-Nielsen 1994, Pott \& Pott 2000).

4. Echinodorus tenellus (Mart. ex Schult. \& Schult. f.) Buchenau, Abh. Naturwiss. Vereines Bremen 2:21. 1868. $\equiv$ Alisma tenellum Mart. ex Schult. \& Schult. f., Syst. veg. 7(2):1600. 1830. Type: BRAZIL: Buritihaes, Contendas, C.F.P. Martius a 56 (lectotype M, fide Rataj 1975).

Figure 2E.

Plants delicate, annual, glabrous, stoloniferous, to $6 \mathrm{~cm}$ tall. Submersed leaves present, $5 \mathrm{~cm}$ long, $0.2 \mathrm{~cm}$ wide, with a sheathing base $2 \mathrm{~cm}$ long; emersed leaves petiolate, $1.5-4.5 \mathrm{~cm}$ long, $0.2 \mathrm{~cm}$ wide, the blade linearlanceolate with a sheathing base $1 \mathrm{~cm}$ long, pellucid markings absent. Inflorescence umbelliform with only one floral whorl, erect, up to $6 \mathrm{~cm}$ long, without vegetative proliferations. Bracts triangular, basally united, coarse with delicate margins, $0.3 \mathrm{~cm}$ long, $0.1-0.2 \mathrm{~cm}$ wide. Flowers perfect, 2-28, 0.6-1 cm diam.; sepals appressed, $0.3 \mathrm{~cm}$ long; petals clawed, $0.2-0.4 \mathrm{~cm}$ long, 0.1-0.3 cm wide; stamens 9, $1 \mathrm{~cm}$ long, carpels 15-20. Fruits not observed.

Common name: "pigmy chain-sword", "dwarf Amazonian sword plant", "erva do pântano" (Haynes \& Holm-Nielsen 1994, Pott \& Pott 2000). 
Specimens examined: BRAZIL: GoIÁs: Estrada Jussara-Aragarças, a $100 \mathrm{~km}$ de Jussara, 26-V-1997 (fl.), C.P. Bove et al. 161 (R); 9-X-1997 (fl.), C.P. Bove et al. 240 (R); Estrada Jussara-Britânia a $59 \mathrm{~km}$ de Jussara, 14-XI-1999 (fl.), C.P. Bove et al. 581 (R); Estrada Jussara-Britânia (via Jacilândia), a $59 \mathrm{~km}$ de Jussara, 12-XI-1999 (fl.), C.P. Bove et al. 550 (R, SPF);
Estrada para Peixe (GO-334), a $21 \mathrm{~km}$ da GO-164, 16-XI-1999 (fl.), C.P. Bove et al. 622 (R, SPF). МАто Grosso: Estrada Água Boa-Cocalinho, a $58 \mathrm{~km}$ de Água Boa, 11-X-1997 (fl.), C.P. Bove et al. 255 (R, SPF).

Habitat: amphibious or submersed plant, growing in still water up to $50 \mathrm{~cm}$ deep or in muddy soil. Specimens collected in dry soil were intensively

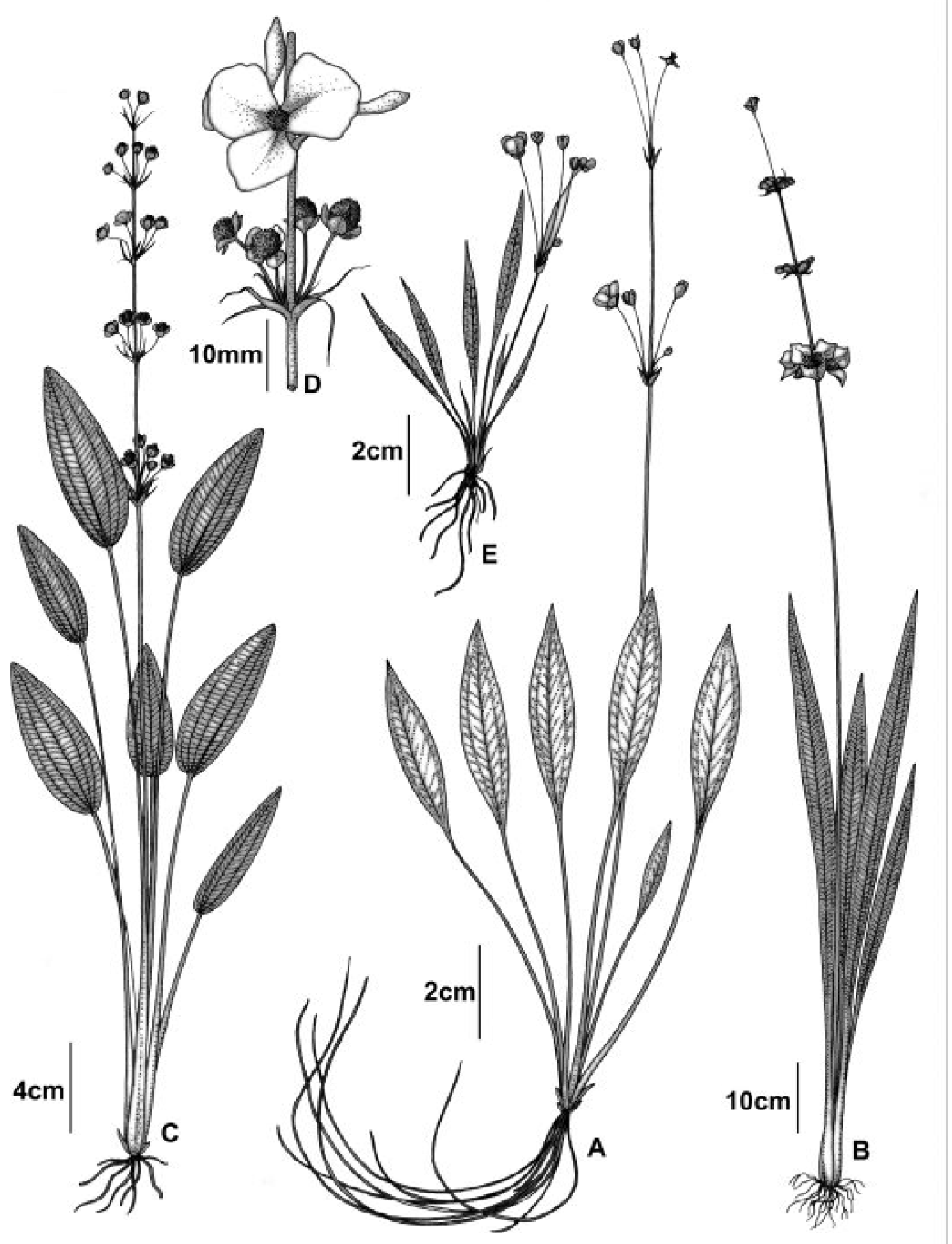

Figure 2. Species of Echinodorus from the upper and middle Araguaia basin. A. E. bolivianus, habit. B. E. longipetalus, habit. C. E. subalatus, habit. D. E. subalatus, flower. E. E. tenellus, habit (A: Bove et al. 238, B: Bove et al. 158, C-D: Bove et al. 264, E: Bove et al. 255). 
flowering compared to flowering specimens found in wet environments. Frequent and usually locally abundant.

Distribution: northeastern U.S.A. to southern Brazil, states of Amazônia, Bahia, Minas Gerais, Goiás, Mato Grosso, Mato Grosso do Sul, Minas Gerais, Pará, Rio de Janeiro, Roraima, Santa Catarina, São Paulo (Haynes \& Holm-Nielsen 1994, Pott \& Pott 2000).

5. Sagittaria guayanensis Kunth subsp. guayanensis, Nov. gen. sp. 1:250. 1816. Type: COLOMBIA: Guainia, Humboldt s.n. (holotype P?; fragment at MO; lectotype TCD, Rataj 1972; isolectotypes LE, W).

Figure 3A-B.

Plants perennial, glabrous or pubescent, to $50 \mathrm{~cm}$ tall. Stems present as corms. Submersed leaves present, $0.5-1 \mathrm{~cm}$ long; emersed leaves floating, the peciole to $30 \mathrm{~cm}$ long, with a sheathing base to $10 \mathrm{~cm}$ long, the blade sagittate, $1-4 \mathrm{~cm}$ long, $0.5-5 \mathrm{~cm}$ wide, pellucid markings absent. Inflorescence racemose, floating, rarely erect, 1-20 cm long, with 1-5 whorls, 3 flowers each, without vegetative proliferations. Bracts linearlanceolate, free, delicate, $0.3-0.5 \mathrm{~cm}$ long. Flowers imperfect; sepals erect, $0.5-1 \mathrm{~cm}$ long, $0.5 \mathrm{~cm}$ wide; petals clawed, $0.5-1 \mathrm{~cm}$ long, $0.6 \mathrm{~cm}$ wide; male flowers with sterile carpels, stamens $6,0.3 \mathrm{~cm}$ long; female flowers with a ring of sterile stamens, carpels numerous. Fruits oblanceolate, keeled, without glands, the margins echinate, $0.2 \mathrm{~cm}$ long, the beak lateral, erect.

Common name: "lagartixa", "largatissa", "corazón de agua" (Pott \& Pott 2000).

Specimens examined: BRAZIL: GoIÁs: Estrada Araguapaz-Aruanã, a 11 Km de Araguapaz, 13-X-1997 (fl., fr.), C.P. Bove et al. 281 (R, SPF); 13-X-1997 (fl., fr.), C.P. Bove et al. 282 (R); Estrada JussaraAragarças, a $100 \mathrm{~km}$ de Jussara, 25-V-1997 (fl.), C.P. Bove et al. 136, fl. (R); 25-V-1997 (fl.), C.P. Bove et al. 150 (R); 26-V-1997 (fl.), C.P. Bove et al. 187 (R); 26-V-1997 (fl.), C.P. Bove et al. 189 (R); 27-V-1997 (fl., fr.), C.P. Bove et al. 193 (R, SPF); Estrada Jussara-Aragarças, a $97 \mathrm{~km}$ de Jussara, 27-V-1997 (fl.), C.P. Bove et al. 196 (R); 11-XI-1999 (fl., fr.), C.P. Bove et al. 513 (R); Estrada JussaraBritânia a 39,6 km de Jussara, 12-XI-1999 (fl., fr.), C.P. Bove et al. 561 (R, SPF); Estrada JussaraBritânia, a 54,4 km de Jussara, 28-V-1997 (fl., fr.), C.P. Bove et al. 200 (R, SPF); Estrada JussaraBritânia (via Jacilândia), a 78,2 km de Jussara, 12-XI-1999 (fl.), C.P. Bove et al. 536 (R). МАто Grosso: Estrada Paranatinga-Canarana km 151, 12-II-1993 (fl., fr.), C.P. Bove et al. 95 (R).
Habitat: floating-leaved plant, growing in still water $50-100 \mathrm{~cm}$ deep. A very common species in the Araguaia river region, usually locally abundant.

Distribution: from southern U.S.A. to Argentina and Brazil, states of Acre, Amazonas, Ceará, Goiás, Maranhão, Mato Grosso, Mato Grosso do Sul, Minas Gerais, Pará, Piauí, Rondônia and Roraima (Haynes \& Holm-Nielsen 1994, Pott \& Pott 2000).

6. Sagittaria rhombifolia Cham., Linnaea 10:219. 1835. Type: BRAZIL: s. loc., Sellow s.n. (lectotype E, fide Rataj 1972; isolectotype K, LE).

Figure 3C-E.

Plants perennial, glabrous, $50-100 \mathrm{~cm}$ tall. Submersed leaves present, ca. $50 \mathrm{~cm}$ long, $0.5 \mathrm{~cm}$ wide; emersed leaves erect, petiolate, $10-45 \mathrm{~cm}$ long, with a sheathing base to $5-10 \mathrm{~cm}$ long, the blade linearlanceolate, $5-15 \mathrm{~cm}$ long, $0.2-2.3 \mathrm{~cm}$ wide, pellucid markings absent. Inflorescence racemose, erect, rarely floating, 20-40 cm long, with 2-10 whorls with 3 flowers each, without vegetative proliferations. Bracts lanceolate, united at base, delicate to coarse, $0.5-2.5 \mathrm{~cm}$ long. Flowers imperfect; male flowers with spreading pedicels, 2-4 cm long, sepals erect, $1 \mathrm{~cm}$ long, $0.5 \mathrm{~cm}$ wide; petals clawed, $2 \mathrm{~cm}$ long, $1 \mathrm{~cm}$ wide; stamens 9-12, $0.5 \mathrm{~cm}$ long, sterile carpels present; female flowers with erect pedicels, spreading and enlarged in fruit, $1.5-2 \mathrm{~cm}$ long, appressed in flower and fruit, $1.5-2 \mathrm{~cm}$ long, $1-2 \mathrm{~cm}$ wide, petals clawed, $1.5-3 \mathrm{~cm}$ long, $0.5 \mathrm{~cm}$ wide, without sterile stamens, carpels numerous. Fruits oblanceolate, keeled, without glands, $0.1 \mathrm{~cm}$ long, the beak lateral, horizontal.

Common name: "lagartixa" (Pott \& Pott 2000).

Specimens examined: BRAZIL: GoIÁs: Estrada Jussara-Britânia, a 62 km de Jussara, córrego Píndaíba, 14-XI-1999 (fl.), C.P. Bove et al. 582 (R); Estrada Jussara-Aragarças, a $100 \mathrm{~km}$ de Jussara, 25-V-1997 (fl.), C.P. Bove et al. 143 (R); 25-V-1997 (fl., fr.), C.P. Bove et al. 144 (R); 25-V-1997 (fl.), C.P. Bove et al. 145 (R); 25-V-1997, C.P. Bove et al. 146 (R); 25-V-1997 (fl.), C.P. Bove et al. 147 (R); 25-V-1997 (fl.), C.P. Bove et al. 148 (R); 25-V-1997 (fl.), C.P. Bove et al. 149 (R); 26-V-1997 (fl., fr.), C.P. Bove et al. 162 (R); 26-V-1997 (fl.), C.P. Bove et al. 163 (R); 26-V-1997 (fl., fr.), C.P. Bove et al. 164 (R); 26-V-1997 (fl.), C.P. Bove et al. 165 (R); 9-X-1997 (fl.), C.P. Bove et al. 236 (R, SPF); Estrada JussaraAragarças, a $111 \mathrm{~km}$ de Jussara, 11-XI-1999 (fl., fr.), C.P. Bove et al. $526(\mathrm{R})$; Estrada Jussara-Britânia (via Jacilândia), a 42,2 km de Jussara, 12-XI-1999 (fl.), C.P. 
Bove et al. 560 (R); Estrada Caiapônia-Iporã, a 54 km do trevo, 19-XI-99 (fl., fr.), C.P. Bove et al. 670 (R, SPF). Mato Grosso: Paranatinga, $39 \mathrm{~km}$ ao sul, 9-II-1993 (fl., fr.), C.P. Bove et al. 89 (R).

Habitat: emergent plant, growing in still or running water $50-100 \mathrm{~cm}$ deep. A very common species in this region.

Distribution: from southern Costa Rica to Argentina. In Brazil in the states of Bahia, Distrito Federal, Mato Grosso, Mato Grosso do Sul, Minas Gerais, Pará, Paraná, Piauí, Rio de Janeiro, Santa Catarina and São Paulo (Haynes \& Holm-Nielsen 1994, Pott \& Pott 2000).

7. Hydrocleys nymphoides (Willd.) Buchenau, Index Crit. Butom. Alism. Juncag. p. 9. 1868. $\equiv$ Stratiotes nymphoides Willd., Sp. pl. 4(2):821. 1806. Type: VENEZUELA: s. loc., Humboldt \& Bonpland s.n. (holotype B-W).

Figure 4A-C.
Plants to $50 \mathrm{~cm}$ tall, stoloniferous. Floating leaves 7-20 cm long, 2-6 cm wide, with an ovate-orbicular blade, petiolate, with a sheathing base $5 \mathrm{~cm}$ long. Inflorescences with 1-5 flowers, scape 5-10 cm long. Bracts elliptic, $1-2 \mathrm{~cm}$ long, $0.5 \mathrm{~cm}$ wide. Sepals with midvein inconspicuous and hialine margins, $3 \mathrm{~cm}$ long, $1 \mathrm{~cm}$ wide; petals reflexed, pale yellow to white with a yellow base, $2.5 \mathrm{~cm}$ long, $3 \mathrm{~cm}$ wide; stamens $20-30$ in 2 series or more, $1 \mathrm{~cm}$ long, staminodes numerous; carpels 7, $1.5 \mathrm{~cm}$ long. Fruits not observed.

Common name: "lagartixa", "lagartissa", "amapolade-água”, "water poppy" (Pott \& Pott 2000).

Specimens examined: BRAZIL: Mato Grosso: Estrada Mozarlândia-Nova Crixás km 18, 13-X-1997 (fl.), C.P. Bove et al. 276 (R, SPF); Estrada Mozarlândia-Nova Crixás a 18,6 km do trevo, 15-XI-1999 (fl.), C.P. Bove et al. 606 (R, SPF).

Observations: flowers closed early in the morning, opening around 10:00 am.

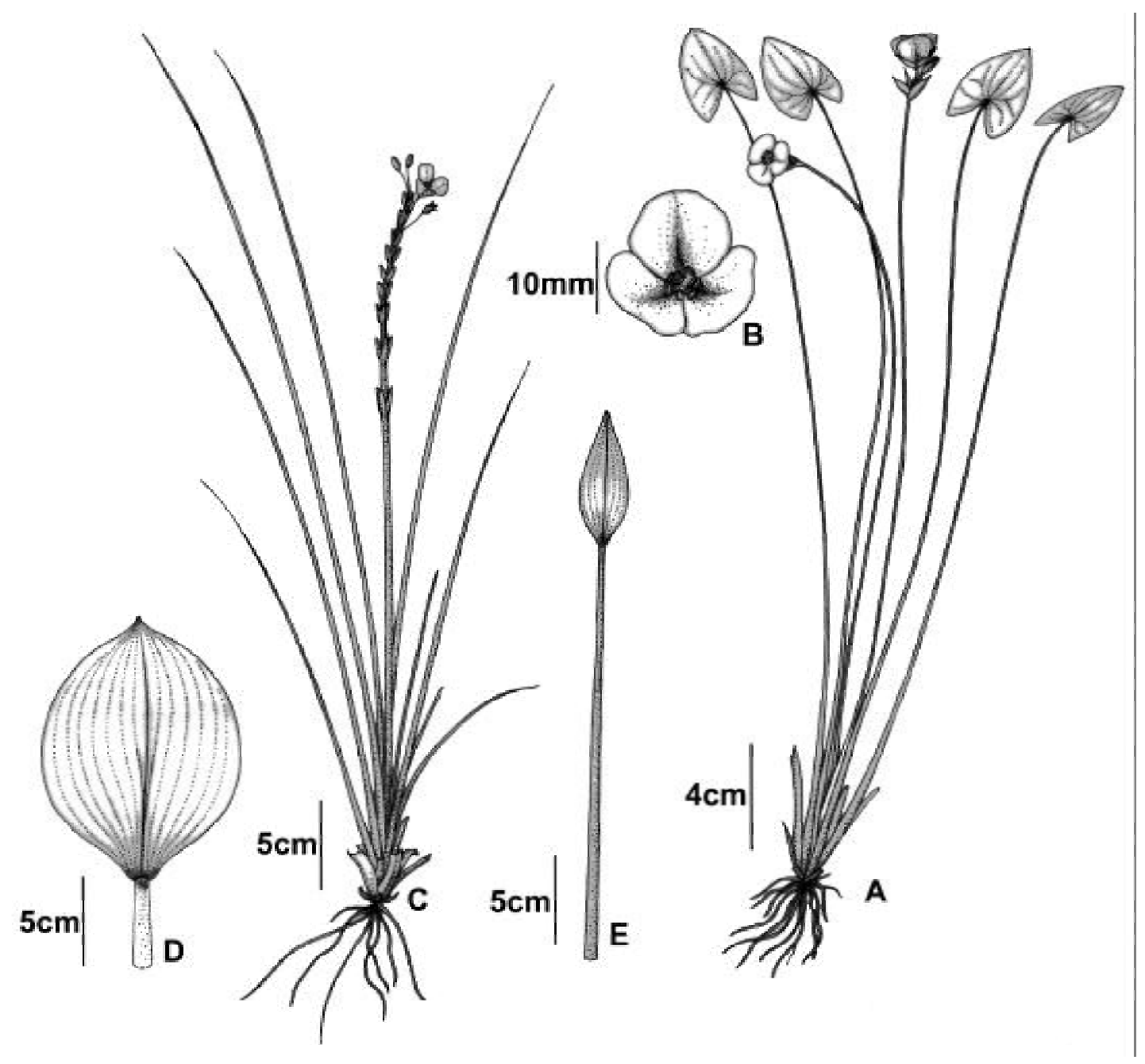

Figure 3. Species of Sagittaria from the upper and middle Araguaia basin. A-B. S. guayanensis subsp. guayanensis. A. Habit; B. Male flower. C-E. S. rhombifolia. C. Habit; D-E. Leaves (A-B: Bove et al. 200, C-D: Bove et al. 148, E: Bove et al. 670). 

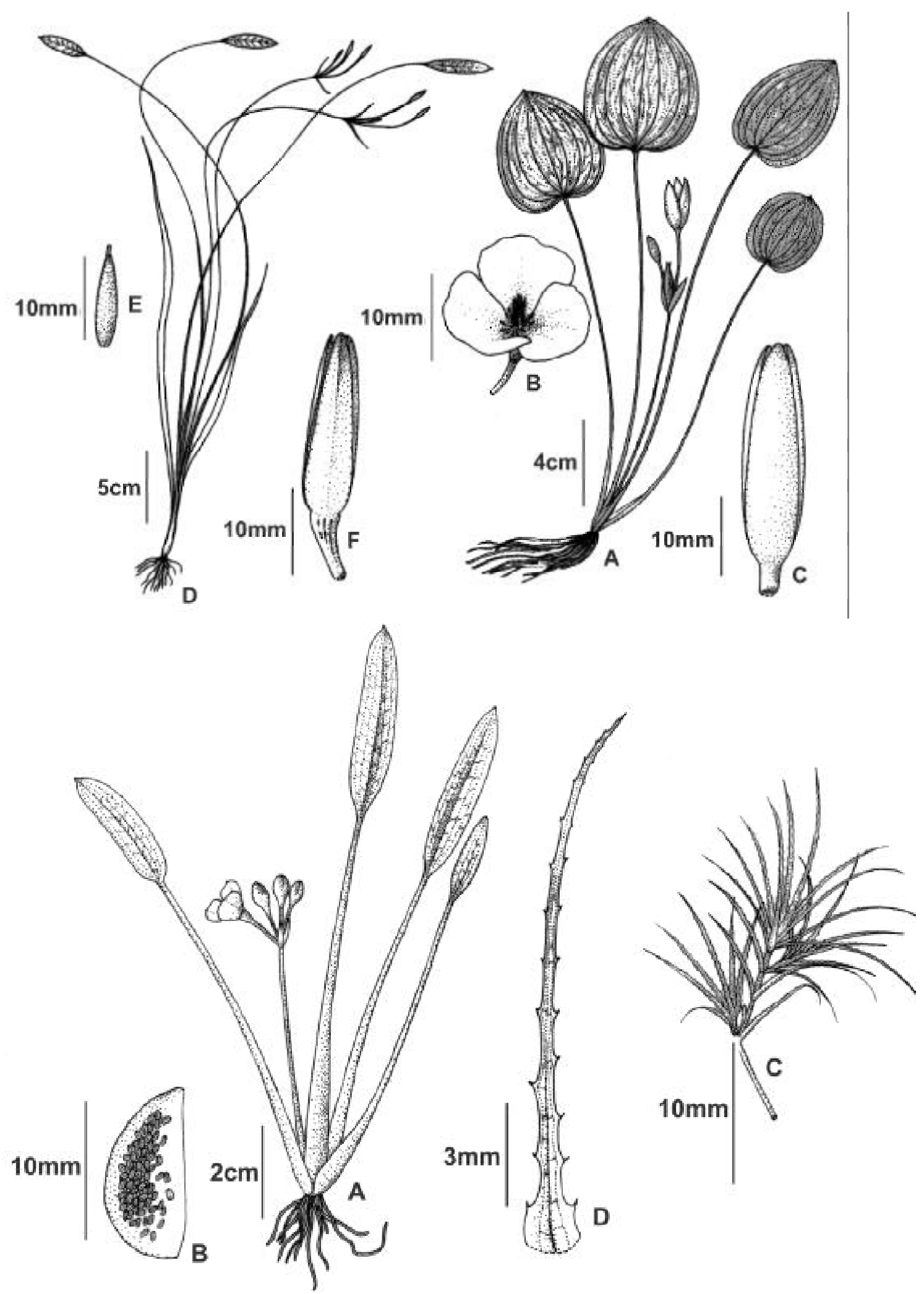

Figure 4. Species of Alismataceae and Najadaceae from the upper and middle Araguaia basin. A-C. Hydrocleys nymphoides. A. Habit; B. Flower; C. Calix. D-F. Hydrocleys parviflora. D. Habit; E. Fruit; E. Calix. G-H. Limnocharis laforestii. G. Habit; H. Fruit. I-J. Najas microcarpa. I. Habit; J. Leaf. (A-C: Bove et al. 276, D-F: Bove et al. 201, G-H: Bove et al. 618, I-J: Bove et al. 260). 
Habitat: plants with floating leaves, growing in still water 50-100 cm deep. Sporadic.

Distribution: tropical and subtropical North America, Central America, and South America. In Brazil in the states of Bahia, Ceará, Mato Grosso, Mato Grosso do Sul, Pará, Paraíba, Pernambuco, Piauí, Rio Grande do Norte, Rio de Janeiro and Santa Catarina (Haynes \& Holm-Nielsen 1992, Pott \& Pott 2000).

8. Hydrocleys parviflora Seub. in Mart. \& Eichler, Fl. bras. 3(1):117. 1847. Type: BRAZIL: s. loc., Martius s.n. (lectotype M, fide Haynes \& Holm-Nielsen 1992). Figure 4D-F.

Plants to $100 \mathrm{~cm}$ tall, stoloniferous. Floating leaves with an elliptic blade, $5 \mathrm{~cm}$ long, $1 \mathrm{~cm}$ wide, petiole up to $100 \mathrm{~cm}$ long with a sheathing base $10 \mathrm{~cm}$ long. Inflorescences with 2-10 flowers, scape $100 \mathrm{~cm}$ long. Bracts lanceolate, $2 \mathrm{~cm}$ long, $1 \mathrm{~cm}$ wide. Sepals with conspicuous midvein, $2 \mathrm{~cm}$ long, $1 \mathrm{~cm}$ wide; petals white, $1.5 \mathrm{~cm}$ long, $1 \mathrm{~cm}$ wide; stamens 5 in one whorl, $0.3 \mathrm{~cm}$ long, staminodes 3; carpels 3, $0.4 \mathrm{~cm}$ long. Fruits $1.2 \mathrm{~cm}$ long, beak $0.1 \mathrm{~cm}$ long; seeds numerous, glandular pubescent, $1 \mathrm{~mm}$ long.

Common name: "lagartixa" (Pott \& Pott 2000).

Specimens examined: BRAZIL: GoIÁs: Estrada Jussara-Britânia, a 54 km de Jussara, 28-V-1997 (fl.), C.P. Bove et al. 201 (R, SPF).

Habitat: plants with floating leaves, growing in still water 50-100 cm deep. Rare in the Araguaia river region.

Distribution: southern Mexico to Costa Rica, northern Colombia, Venezuela and Bolivia to east-central Brazil, in the states of Goiás, Mato Grosso do Sul, Minas Gerais and Rio de Janeiro (Haynes \& Holm-Nielsen 1992).

9. Limnocharis laforestii Griseb., Bonplandia 6(1):11. 1858. Type: PANAMA: s. loc. Duchassaing et al. s.n. (lectotype GOET, fide Novelo 1987; isolectotype GOET).

Figure 4G-H.

Plants to $50 \mathrm{~cm}$ tall, rhizomatous. Leaves erect, petiolate, with an elliptic to ovate blade, $15-25 \mathrm{~cm}$ long, $1-2 \mathrm{~cm}$ wide with a sheathing base $5 \mathrm{~cm}$ long. Inflorescences with up to 5 flowers. Bracts elliptic-linear,
0.5-1.5 cm long, 0.3-1 cm wide; pedicels $1 \mathrm{~cm}$ long, $1 \mathrm{~cm}$ wide. Sepals ovate, $1 \mathrm{~cm}$ long, $0.7 \mathrm{~cm}$ wide; petals yellow, ovate to orbicular, $1.5 \mathrm{~cm}$ long, $0.8 \mathrm{~cm}$ wide; stamens 15-20, $0.6 \mathrm{~cm}$ long, staminodes numerous; carpels 20-30, $0.4 \mathrm{~cm}$ long. Fruits $0.1 \mathrm{~cm}$ long; seeds numerous, $0.1 \mathrm{~cm}$ long.

Common name: "camalote" (Pott \& Pott 2000).

Specimens examined: BRAZIL: GoIÁs: Estrada Britânia-Aruanã, a 5 km de Jussara, 27-V-1997 (fl., fr.), C.P. Bove et al. 205 (R). Mато Grosso: Estrada Água Boa-Cocalinho, a 144 km da GO-164, 12-X-1997 (fl., fr.), C.P. Bove et al. 268 (R, SPF).

Observations: the specimens examined presented the peduncles shorter than the petioles and the pedicels not conspicuously winged. The staminodes were numerous, like in Limnocharis flava (L.) Buch. The number of staminodes, however, is not considered a diagnostic character according to the last taxonomic treatment available for the genus (Haynes \& HolmNielsen 1992).

Habitat: emergent plant, growing in still water 50-100 cm deep. Sporadic.

Distribuição: Mexico to Bolivia, Argentina and Brazil, in the states of Bahia, Goiás, Maranhão, Mato Grosso, Mato Grosso do Sul, Paraíba, and Piauí (Haynes \& Holm-Nielsen 1992, Pott \& Pott 2000).

\section{Hydrocharitaceae Juss.}

Herbaceous, annual or perennial, glabrous, not lactiferous. Stems erect, rhizomatous or stoloniferous, elongated or contracted. Leaves submersed, floating or partially emersed, basal, alternate or whorled, sessile or petiolate, margins entire or serrulate, the blade linear to eliptic-lanceolate, 1- or pluriveined, without pellucid markings. Inflorescences complex with 1-many flowers or flowers solitary, with subtending spathe, composed of 1-2 free or united bracts. Flowers epigynous, perfect or imperfect, perianth actinomorphic, of 6 free segments in 2 series: sepals 3, green, persistent; petals 3, larger than sepals, white or yellow, delicate, deciduous; stamens 3 , 6 or 9, free; carpels 3, fused, ovary 1-loculed, prolongated in a free hypanthium, numerous ovules of parietal or laminar placentation, the styles flattened. Fruit a capsule with numerous fusiform seeds.

Key to the species of Hydrocharitaceae of the upper and middle Araguaia river region

1. Stems elongated; leaves sessile; flowers white

2. Flowers perfect; stamens 3

10. Apalanthe granatensis

2. Flowers imperfect; stamens 9 11. Egeria heterostemon

1. Stems contracted; leaves petiolate; flowers yellow 12. Ottelia brasiliensis 
10. Apalanthe granatensis (Bonpl.) Planch., Ann. Mag. Nat. Hist., Sér. 2 (1):87. 1848. $\equiv$ Elodea granatensis Bonpl. in Humbolt \& Bonpland, Pl. aequinoct. 2(16):150, pl.128. 1813. Type: COLOMBIA: "in aquis Novas Granatea, prope urbem Guaduas", "entre Honda et Cune", elev. 1,150 m, Humboldt \& Bonpland s.n. (holotype, plate in Humbolt \& Bonpland, Pl. aequinoct. 2 (16), pl. 128. 1813).

Figure 5A-B.

Plants submersed or partially emersed. Stems elongated, terete, irregularly branched. Prophylls paired at the base of branches, deltate; leaves alternate or whorled, sessile, 3-7 in each node, margins serrulate, 1-veined, the blade linear and gradually attenuated at the apex, 1-1.5 cm long. Subtending spathe consisting of two bracts, sessile, axillary, urn-shaped, $0.5 \mathrm{~cm}$ long, $0.1 \mathrm{~cm}$ wide. Flowers solitary, perfect, sessile, emersed; sepals ovate, reflexed, $0.2 \mathrm{~cm}$ long, $0.1 \mathrm{~cm}$ wide; petals obovate, white, delicate, $0.3 \mathrm{~cm}$ long, $0.2 \mathrm{~mm}$ wide; stamens 3, $0.2 \mathrm{~cm}$ long; styles 3, flattened, 2- or 3-divided, with nectaries at the base, $0.2 \mathrm{~cm}$ long; ovary $0.5 \mathrm{~cm}$ long, attenuate above in an elongated free hypanthium to $5 \mathrm{~cm}$ long. Capsule ellipsoidal, irregularly dehiscent, $0.5 \mathrm{~cm}$ long; seeds up to 30 , ca. $1 \mathrm{~mm}$ long, covered with hairs.

Common name: "lodinho branco" (Pott \& Pott 2000).

Specimens examined: BRAZIL: GoIÁs: Estrada Aruanã-Goiás 8,8 km de Aruanã, 29-V-1997 (fl.), C.P. Bove et al. 224 (R, RB); Estrada GO-334, a 47,8 km da GO-164, 16-XI-1999 (fl.), C.P. Bove et al. 632 (R); Brasilândia, Tupiratins, Rio Feio, 20-III-1976 (fl.), G. Hatschbach \& R. Kummrov 38497 (MBM). Мато Grosso. Estrada Água Boa-Cocalinho, a $148 \mathrm{~km}$ de Água Boa, 12-X-1997 (fl., fr.), C.P. Bove et al. $273 b$ (R, RB); Estrada Água Boa-Rio das Mortes, 20-II-1993, C.P. Bove et al. 114 (R, CPAP); Córrego do Rosário, Forquilha do Rio, III-1918 (fr.), J.G. Kuhlmann 1620 (R).

Habitat: submersed plant, growing in still or running water up to $50 \mathrm{~cm}$ deep. Sporadic.

Distribution: tropical South America, in Brazil in the states of Amazonas, Bahia, Goiás, Mato Grosso, Mato Grosso do Sul, Maranhão, Minas Gerais, Pará, Pernambuco, Piauí, Rio de Janeiro, Roraima, São Paulo (Cook 1985).

11. Egeria heterostemon S. Koehler \& C.P. Bove, Novon 11:63. 2001. Type: BRAZIL: GoIÁs, Estrada AruanãGoiânia, 19 km from Aruanã, C.P. Bove et al. 225 (holotype RB; isotypes CPAP, HUEFS, MBM, MO, R). Figure 5C-I.
Plants submersed or partially emersed. Stems elongated, terete, irregularly branched. Prophylls paired, deltate; leaves opposite or whorled, sometimes alternate near the base, sessile, 4-10 in each node, frequently recurved, linear, gradually attenuated to an acute apex, 0.6-0.8 cm long, margins and apex bearing unicellular, thick-walled prickle-hairs. Intravaginal squamules usually 2 in each leaf axil, ovoid to orbicular, 0.1-0.2 mm long. Spathe sessile, axillary, ovoid, $1.0 \mathrm{~cm}$ long, with 2 apical teeth. Male flowers: 2 in each spathe, rarely more, pedicels up to $4.0 \mathrm{~cm}$ long, emersed; sepals ovate and boat-shaped, $0.2 \mathrm{~cm}$ long, $0.1 \mathrm{~cm}$ wide; petals suborbicular, white, delicate, $0.2-0.4 \mathrm{~cm}$ long, $0.2-0.3 \mathrm{~mm}$ wide; stamens 9 , in alternate whorls of 3; outer and median whorls $0.1 \mathrm{~cm}$ long; filaments glabrous; stamens in the outer whorl usually smaller than those of median whorl, although similar in form. Inner whorl: $0.15 \mathrm{~cm}$ long, filaments strongly pilose distally; nectary 1 , central, 3-lobed, $0.2 \mathrm{~mm}$ wide. Female flower: 1 in each spathe, sessile, emersed; sepals ovate and boat-shaped, $0.2 \mathrm{~cm}$ long, $0.1 \mathrm{~cm}$ wide; petals suborbicular, white, delicate, $0.3 \mathrm{~cm}$ long, $0.2 \mathrm{~mm}$ wide; staminodes 3 , strongly papillose distally; $0.15 \mathrm{~cm}$ long, styles 3 , two-thirds divided along length; ovary up to $0.3 \mathrm{~cm}$ long, ovoid, attenuate above in an elongated free hypanthium to $0.35 \mathrm{~cm}$ long. $30-40$ ovules with parietal placentation. Fruit unknown.

\section{Common name: unknown}

Specimens examined: BRAZIL: GoIÁs: Estrada Aruanã-Goiás, 19 km de Aruanã, 29-V-1997 (fl.), C.P. Bove et al. 225 (CPAP, HUEFS, MBM, MO, R, RB); Mato Grosso: Estrada Água Boa-Cocalinho, a 148 km de Água Boa, 12-X-1997 (f1.), C.P. Bove et al. $273 a$ (R, RB).

Habitat: submersed plant, growing in still or running water up to $50 \mathrm{~cm}$ deep along stream margins and in swampy environments. Rare.

Distribution: Median Araguaia river in the states of Mato Grosso and Goiás (Koehler \& Bove 2001).

12. Ottelia brasiliensis (Planch.) Walp., Ann. Bot. Syst. 3(3):510. 1852. 三 Damasonium brasiliensis Planch., Ann. Sci. Nat. Bot. (Paris) 11:81, t.14. 1849. Type: BRAZIL: Minas GeRAIs, Gardner 5191 (holotype K). Figure 5J-K.

Plants submersed. Stems contracted, rhizomatous. Juvenile leaves linear, sessile, ephemerous; adult leaves petiolate, up to $50 \mathrm{~cm}$, with a sheathing base, blade membranaceous, elliptic-lanceolate, margins entire or undulate, $10-40 \mathrm{~cm}$ long, $3-5 \mathrm{~cm}$ wide. Subtending spathe 

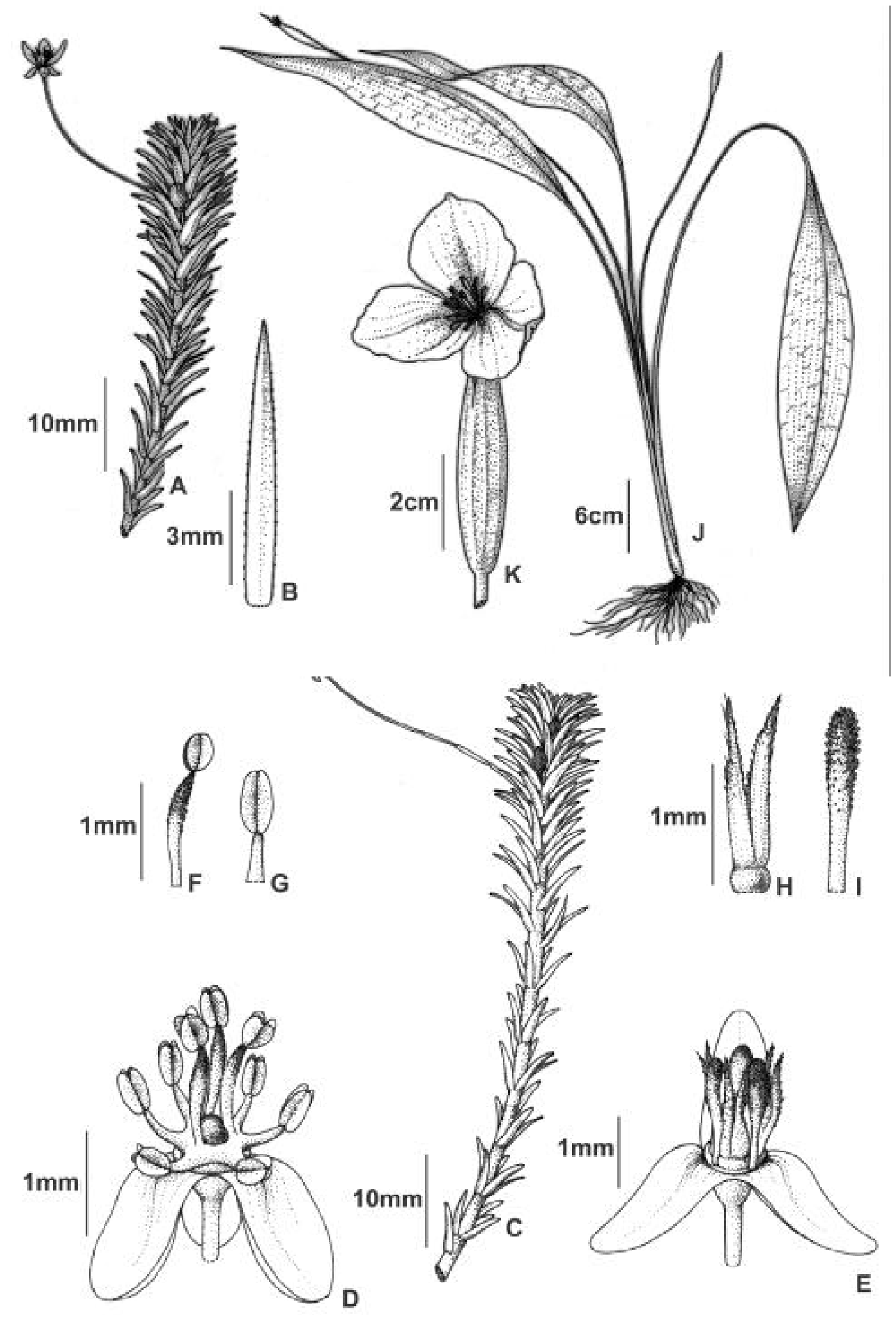

Figure 5. Species of Hydrocharitaceae from the upper and middle Araguaia basin. A-B. Apalanthe granatensis. A. Habit; B. Leaf. C-I. Egeria heterostemon. C. Habit; D. Male flower; E. Female flower; F. Stamen of outer whorl; G. Stamen of inner whorl; H. Style; I. Staminode. J-K. Ottelia brasiliensis. J. Habit. K. Flower. (A-B: Bove et al. 114, C-I: Bove et al. 225, reprodution of original publication in Novon, J-K: Bove et al. 194). 
consisting of two bracts, emersed, membranaceous, urn-shaped, $5 \mathrm{~cm}$ long, $1 \mathrm{~cm}$ wide. Flowers solitary, perfect, pedicels $2 \mathrm{~cm}$ long; sepals obovate-lanceolate, with delicate margins, $1-2 \mathrm{~cm}$ long, $0.5 \mathrm{~cm}$ wide; petals obovate-orbicular, yellow, delicate, margins undulate, $2 \mathrm{~cm}$ long, $2 \mathrm{~cm}$ wide; stamens $6,1 \mathrm{~cm}$ long; styles 3 , flattened, linear, 2- or 3-divided, $3 \mathrm{~cm}$ long, with nectaries at the base, ca. $0.2 \mathrm{~cm}$ long; ovary $0.5 \mathrm{~cm}$ long, anthers elongated with a mucronate connective. Fruits not observed.

Specimens examined: BRAZIL: GoIÁs: Estrada Jussara-Aragarças, a $100 \mathrm{~km}$ de Jussara, 27-V-1997 (fl.), C.P. Bove et al. 191 (R, SPF); 9-X-1997 (fl., fr.), C.P. Bove et al. 239 (R, SPF); Estrada Jussara-Aragarças, a $97 \mathrm{~km}$ de Jussara, 27-V-1997 (fl.), C.P. Bove et al. 194 (R); 11-XI-1999 (fl.), C.P. Bove et al. 503 (R, SPF); Estrada Jussara-Britânia, via Jacilândia, a 42 km de Jussara, 12-XI-1999 (fl.), C.P. Bove et al. 556 (R); Estrada Jussara-Britânia, via Jacilândia, a 59 km de Jussara, 12-XI-1999 (fl.), C.P. Bove et al. 551 (R).

Habitat: submersed plant growing in running water 50-100 cm deep. Sporadic.

Distribution: southeastern Brazil, in the states of Minas Gerais, Mato Grosso, Mato Grosso do Sul, and Paraná.

\section{Najadaceae Juss.}

Herbaceous, submersed, glabrous, not lactiferous. Stems elongated with short to long internodes, irregularly branched, smooth to spinulose, easily fragmented. Leaves alternate to subopposite or apparently whorled, sessile, sheathed, blade linear to lanceolate, 1-veined, flattened to recurved, margins with inconspicuous to conspicuous, unicellular to multicellular serrulate projections. Flowers hypogynous, imperfect, inconspicuous, solitary or not, perianth absent. Male flowers shortly pedunculate, subtended by an involucral bract, bearing 1 stamen with a sessile anther; female flowers sessile with involucral bract rarely present 1-carpelate, 1-loculed, 1 ovule, stigma 3. Fruit a nutlet. Seeds elliptic, ovate or fusiform, ridged, testa pitted with many aureolae of distinct shapes.

13. Najas microcarpa K. Schum. in Mart., Eichler \& Urban, Fl. bras. 3(3):727. 1894. Type: PARAGUAY: Rio Paraguay, Weddell 3289 (lectotype P).

Figure 4I-J.

Stems elongated with short internodes, smooth, leaves concentrated in the terminal region. Leaves subopposite or apparently whorled, recurved with conspicuous, multicellular serrulate margins, $0.1-0.2 \mathrm{~cm}$ long, and ciliated sheaths bearing microscopic projections. Seeds fusiform to obovate, $0.1-0.2 \mathrm{~cm}$ long, areolate with square to rectangular ornamentations.

Common name: "lodo", "najad" (Pott \& Pott 2000).

Specimens examined: BRAZIL: GoIÁs: Estrada Jussara-Aragarças, a $3 \mathrm{~km}$ de Jussara, 11-XI-1999 (fl., fr.), C.P. Bove et al. 496 (R, SPF). Мато Grosso: Estrada Água Boa-Cocalinho, a $59 \mathrm{~km}$ de Água-Boa, 11-X-1997 (fl., fr.), C.P. Bove et al. 260 (R, SPF).

Habitat: free or attached on the substrate, submersed, growing in a small brackish pond as a dominant species in association with Cabomba furcata Schultes \& Schultes f. Sometimes also captured between rocks of rivers. Sporadic.

Distribution: South America in French Guiana, Venezuela, Colombia, Paraguay and Brazil, in the states of Amazonas, Maranhão, Mato Grosso and Mato Grosso do Sul (Lowden 1986, Pott \& Pott 2000).

Albeit its biological importance, the diversity of tropical aquatic ecosystems remains poorly known (Crow 1993). The elaboration of checklists followed by taxonomic studies of aquatic plants constitute a basic need for Brazilian aquatic ecosystems today, especially in regions that are poorly studied, such as the northern and central regions. Unfortunately Brazilian aquatic ecosystems have been continuously destroyed or altered by the deviation of natural water courses, by local direct and indirect pollution of the water as well as by the destruction of the Cerrado vegetation and Riparian forests, as observed during the development of this study.

Acknowledgments - We are grateful to Wilson Costa for suggesting the exploration of the interesting and still poorly known aquatic flora of the Araguaia basin; to André Gil, Claudio Moreira, Jane Morrey-Jones and Renato Lisbôa for their help in the field and to Eduardo Hicköfel for the illustrations; to the journal Novon (Missouri Botanical Garden Press) for permission to reproduce the illustrations of Egeria heterostemon; and to the curators of the herbaria R, BHCB, CPAP, HUEFS and MBM for sending material for comparative studies. This manuiscript was greatly improved by the comments of Fabio de Barros, Renato Mello-Silva, Robert Haynes, Volker Bittrich, Wilson Costa and an anonymous reviewer. Material was collected with authorization 02001.004264/98-16-AC from Ibama.

\section{References}

ABREU, L.C. 1981. Flora fanerogâmica da reserva do Parque Estadual das Fontes do Ipiranga (São Paulo, Brasil). 102-Alismataceae. Hoehnea 9:119-120. 
ALBUQUERQUE, B.W.P. 1981. Plantas forrageiras da Amazônia. I. Aquáticas flutuantes e livres. Acta Amazonica 11:457-471.

ALMEIDA-REGO, S.C. 1988. Alismataceae Ventenant do Rio Grande do Sul. Dissertação de mestrado, Universidade Federal do Rio Grande do Sul, Porto Alegre.

BERG, M.E.V.D. 1984. Formas atuais de aproveitamento das espécies nativas e exóticas do Pantanal mato-grossense. In Anais do $1^{\circ}$ Simpósio de Recursos Naturais e Sócioeconômicos do Pantanal. Embrapa-DDT-CPAP, Corumbá, p.131-136.

BOVE, C.P., GIL, A.S.B., MOREIRA, C.B.,ANJOS, R.F.B. 2003. Hidrófitas fanerogâmicas de ecossistemas aquáticos temporários da planície costeira do estado do Rio de Janeiro, Brasil. Acta Botanica Brasilica 17:119-135.

COOK, C.D.K. 1985. A revision of the genus Apalanthe (Hydrocharitaceae). Aquatic Botany 21:157-164.

COOK, C.D.K. 1996. Water plants of the world. SPB Academic Publishing, Amsterdam.

COOK, C.D.K. \& URMI-KÖNIG, K. 1984a. Arevision of the genus Egeria (Hydrocharitaceae). Aquatic Botany 19:73-96.

COOK, C.D.K. \& URMI-KÖNIG, K. 1984b. Arevision of the genus Ottelia (Hydrocharitaceae). 2. The species of Eurasia, Australasia and America. Aquatic Botany 20:131-177.

CROW, G.E. 1993. Species diversity in aquatic angiosperms: latitudinal patterns. Aquatic Botany 44:229-258.

ESTEVES, F.A. 1988. Fundamentos de Limnologia. Editora Interciência, Finep, Rio de Janeiro.

ESTEVES, F.A. \& CAMARGO, A.F.M. 1986. Sobre o papel das macrófitas aquáticas na estocagem e ciclagem dos nutrientes. Acta Limnologica Brasiliensia 1:273-298.

FARIA, A.D. 1998. O gênero Eleocharis R. Br. (Cyperaceae) no Estado de São Paulo. Dissertação de mestrado, Universidade Estadual de Campinas, Campinas.

GUIMARÃES, E.S. 1999. Alismataceae da região sul do Pantanal Mato-grossense. Dissertação de mestrado, Universidade de São Paulo, São Paulo.

HAYNES, R.R. \& HOLM-NIELSEN, L.B. 1985. A generic treatment of Alismatidae in the neotropics with special reference to Brazil. ActaAmazonica 15 (supl. 1-2):153-193.

HAYNES, R.R. \& HOLM-NIELSEN, L.B. 1992. The Limnocharitaceae. Flora Neotropica Monographs 56:1-32.

HAYNES, R.R. \& HOLM-NIELSEN, L.B. 1994. The Alismataceae. Flora Neotropica Monographs 64:1-112.

HENRIQUES, R.P.B., ARAÚJO, D.S.D., ESTEVES. F.A. \& FRANCO,A.C. 1988. Análise preliminar das comunidades de macrófitas aquáticas da lagoa de Cabiúnas, Rio de Janeiro, Brasil. Acta Limnologica Brasiliensia 3:783-802.

HOEHNE, F.C. 1948. Plantas Aquáticas. Secretaria da Agricultura do Estado de São Paulo, São Paulo.

IBGE. 1989. Geografia do Brasil - região Centro-Oeste. v.1. Fundação Instituto Brasileiro de Geografia e Estatística, Rio de Janeiro.
IRGANG, B.E. \& GASTAL, C.V.S. 1996. Macrófitas Aquáticas da Planície Costeira do RS. Universidade Federal do Rio Grande do Sul, Porto Alegre.

IRGANG, B.E., PEDRALLI, G. \& WAECHTER, J.L. 1984. Macrófitos aquáticos da Estação Ecológica do Taim, Rio Grande do Sul, Brasil. Rossléria 6:395-404.

JOYCE, J.C. 1990. Pratical uses of aquatic weeds. In Aquatic weeds: the ecology and management of nuisance aquatic vegetation (A.H. Pieterse \& K.J. Murphy, eds.). Oxford University Press, Oxford, p.274-291.

JUDD, W.S., CAMPBELL, C.S., KELLOGG, E.A. \& STEVENS, P.F. 1999. Plant systematics. A phylogenetic approach. Sinauer Associates, Sunderland.

KOEHLER, S. \& BOVE, C.P. 2001. Hydrocharitaceae from Central Brazil: a new species of Egeria and a note on Apalanthe granatensis. Novon 11:63-66.

LES, D.H. \& HAYNES, R.R. 1995. Systematics of the subclass Alismatidae: a synthesis of approaches. In Monocotyledons: systematics and evolution (P.J. Rudall, P.J. Cribb, D.F. Cutter \& C.J. Humphries, eds.). Royal Botanical Gardens, Kew, p.353-377.

LOWDEN, R.M. 1986. Taxonomy of the genus Najas L. (Najadaceae) in the Neotropics. Aquatic Botany 24:147-184.

NIERING, W.A. 1985. Wetlands. The Audubon Society, New York.

NOVELO, A. 1987. Lectotipificación de Limnocharis laforestii Duchass. ex Grieseb., (Limnocharitaceae). Boletín de la Sociedad Botánica de México 47:92-93.

PALOMBO, C.R. \& PEREIRA, M.D.B. 1992. Monitoramento de plantas aquáticas por satélite. Ambiente 6:49-53.

PEDERSEN, T.M. \& KLEIN, R.M. 1976. Limnocaritáceas . In Flora Ilustrada Catarinense (R. Reitz, ed.). Herbario Barbosa Rodrigues, Itajaí, fasc. LIMN, p.1-9.

PEDRALLI, G. 1981. A família Mayacaceae Kunth no Rio Grande do Sul, Brasil. Iheringia, série Botânica 28:47-54.

PEDRALLI, G., STEHMANN, J.R., TEIXEIRA, M.C., OLIVEIRA, V.L. \& MEYER, S.T. 1993a. Levantamento da vegetação aquática (macrófitos) na área da EPDA-Peti, Santa Bárbara, MG. Iheringia, série Botânica 43:15-28.

PEDRALLI, G., MEYER, S.T., TEIXEIRA, M.C., OLIVEIRA, V.L. \& STEHMANN, J.R. 1993b. Levantamento dos macrófitos aquáticos e da mata ciliar do Reservatório de Volta Grande, Minas Gerais, Brasil. Iheringia, série Botânica 43:29-40.

POTT, V.J. 1993. A família Lemnaceae S. F. Gray no Pantanal (Mato Grosso e Mato Grosso do Sul), Brasil. Dissertação de mestrado, Universidade Federal do Paraná, Curitiba.

POTT, V.J. \& CERVI, A.C. 1999. A família Lemnaceae no Pantanal (Mato Grosso e Mato Grosso do Sul) Brasil. Revista Brasileira de Botânica 22:153-174.

POTT, V.J. \& POTT, A. 1997. Checklist das macrófitas aquáticas do Pantanal, Brasil. Acta Botanica Brasilica 11:215-227.

POTT, V.J. \& POTT, A. 2000. Plantas Aquáticas do Pantanal. EMBRAPA, Brasília. 
POTT, V.J., BUENO, N.C., PEREIRA, R.A.C., SALIS, S.M. \& VIEIRA, N.L. 1989. Distribuição de macrófitas aquáticas numa lagoa na Fazenda Nhumirim, Nhecolândia, Pantanal, MS. Acta Botanica Brasilica, suplemento 3:135-168.

POTT, V.J., BUENO, N.C. \& SILVA, M.P. 1992. Levantamento florístico e fitossociológico de macrófitas aquáticas em lagoas da Fazenda Leque, Pantanal, MS. In Anais do VII Congresso da Sociedade Botânica de São Paulo. Sociedade Botânica de São Paulo, Campinas, p.91-99.

PRADO, A.L., HECKMAN, C.W. \& MARTINS, R.F. 1994. The seasonal sucession of biotic communities in wetlands of tropical wet-and-dry climate zone. II. The Aquatic Macrophyte Vegetation in the Pantanal of Mato Grosso, Brasil. 1994. Internationale Revue der Gesamten Hydrobiologie 79:569-589.

RATAJ, K. 1969. Echinodorus longipetalus Mich. and other species with reticulate markings in the blades from Central and South America. Folia Geobotanica \& Phytotaxonomica 4:435-442.

RATAJ, K. 1971. The taxonomy of Echinodorus palaefolius (Nees et Mart.) MacBr. (Alismataceae) and related species from Mexico, Central and South America. Preslia 43:10-61.
RATAJ, K. 1972. Revision of the genus Sagittaria. Part II. The species of West Indies, Central and South America. Annotationes Zoologicae et Botanicae 78:1-61.

RATAJ, K. 1975. Revizion [sic] of the genus Echinodorus Rich. Akvárium-Terarium:1-155.

RATAJ, K. 1978. Alismataceae of Brazil. Acta Amazonica 8 (supl. 1):1-154.

SCHESSL, M. 1997. Flora und Vegetation des nördlichen Pantanal von Mato Grosso, Brazilien. Floristiche Zusammensetzung, Pflanzengesellschaften und Vegetationsdynamic saisonal und permanent überfluteter Standorte eines tropischen Sedimentationbeckens. Martina Galunder-Verlag, Wiel.

TUNDISI, J.G. \& BARBOSA, F.A.R. 1995. Conservation of aquatic ecossystems: present status and perspectives. In Limnology in Brazil (J.G. Tundisi, C.E.M. Bicudo \& T. Matsumura, eds.). ABC/SBL, Rio de Janeiro, p.365-376.

WANDERLEY, M.G.L. 1989. Xyridaceae. In Flora do Estado de Goiás. Coleção Rizzo (J.A. Rizzo, coord.). Universidade Federal de Goiás, Goiânia, v.11, p.1-81. 